\title{
Concussion and Mild Head Injury: Epidemiology, Clinical Considerations and Prognostic Factors
}

José Manuel Ortega Zufiría*, Pilar Jerez Fernández, Jorge Zamorano Fernández, Martin Tamarit Degenhardt, Pedro Poveda Núñez and Remedios López Serrano

Department of Neurosurgery, University Hospital of Getafe, Ctra. de Toledo, Madrid, Spain

*Corresponding author: José Manuel Ortega Zufiria, Department of Neurosurgery, University Hospital of Getafe, Ctra. de Toledo, Madrid, Spain, Tel: +34916839360; Email: fuencarral108@hotmail.com

Rec date: December 28, 2017; Acc date: February 14, 2018; Pub date: February 16, 2018

Copyright: () 2018 Zufiría JMO, et al. This is an open-access article distributed under the terms of the creative commons attribution license, which permits unrestricted use, distribution, and reproduction in any medium, provided the original author and source are credited.

\begin{abstract}
Traumatic brain injury (TBI) is a very usual event, associated with very high morbidity's rate. Moreover, the mild TBI consume a lot of resources, both human and financial. The target of this study was to describe a large series of adult patients suffering mild TBI, treated at the University Hospital of Getafe, between 2010 and 2015 ( $n=2480)$, studying the epidemiological profile and analyzing the diagnosis and treatment, to establish the main prognostic factors that influence the final result. We performed a retrospective study, reviewing medical history. Mild TBI is more common in men, and the most common causative mechanism is the traffic accident, in our region. We propose a model for classifying patients according to risk groups, which divided them into low, intermediate or high risk, which correlates well with the final result. We study the indication of skull radiography and computed tomography (CT), as well as hospital admission for clinical observation.

In this study, the presence of neurological focus on clinical examination, the existence of fracture on plain radiographs, the age and the coagulation disorders are associated with increased likelihood of developing intracranial, and worse prognosis in patients that suffer traumatic injuries. The Glasgow Coma Scale is deficient in determining the outcome of the patient suffering TBI, because do not consider factors such as amnesia or loss of consciousness, very common in patients suffering mild head trauma.
\end{abstract}

Keywords: Craniocerebral trauma; Mild head injury; Epidemiology; Prognosis; Treatment; Radiography; Computed tomography

\section{Introduction}

\section{Concept of cranial traumatism and general considerations}

The incidence and severity of the secondary neurological damage to cranial traumatism differs according to the characteristics of the analyzed population of patients, the harmful mechanisms involved in the trauma and the organization and quality of the sanitary system in that they are handled [1]. In the industrialized countries, the traumatic injuries constitute the first cause of death below 45 years, and the cerebral damage contributes in a decisive way to the fatal result in more than half of the deaths. It has been believed that 70.000 Americans die annually and other 100.000 remain incapacitated in major or less grade as result of a cranial traumatism.

To highlight the magnitude of the problem in epidemiologic terms and prognoses, there is convenient to indicate than in the USA, they register only 11.000 new cases of primary malignant cerebral tumor and 25.000 of hemorrhage subarachnoid per year, respectivament [2]. The information published in Europe, Japan and Australia, they confirm also the transcendence of the cranial trauma in general $[1,2]$. Always has given himself less importance to the light cranial trauma, but this one represents a sanitary problem of enormous interest because a significant number of patients with light trauma will develop potentially mortal complications, which will need urgent medical and neurosurgical performances. Also, most of patients attended by cranial trauma has suffered light trauma, what bears an enormous human and economic effort, and some patients will have persistent symptoms, representing a significant morbidity.

\section{Literature Review}

The severity of the traumatism is undoubtedly the main determinant factor of the final evolution. The classic methods of definition and classification of the coma, based on the duration of the loss of knowledge, anatomic and functional criteria or grade of disconnection of the way, are not quite structured and depend on the subjectivity of the investigator, therefore its clinical application is limited. For the purpose of saving these difficulties, and making easy, objective and trustworthy the observation of the grade of neurological affectation, Jennett and Teasdale introduced the Glasgow Coma Scale (GCS), that values the answer of ocular, verbal opening and the answer motorboat before the external stimuli and is universally accepted [1].

Rimmel and cols. proposed to divide the cranial trauma, according to its severity, in mild (the patient obtains a punctuation between 13 and 15), moderate (the patient obtains a punctuation between 9 and 12) and severe (the patient obtains a punctuation between 3 and 8). Jennett and Bond introduced the scale of Glasgow to value the evolution of the patients who have suffered head injury [1,2].

\section{Epidemiology of the cranial traumatism}

The valuations of incidence of the head injury range enormously according to the countries and used epidemiologic criteria, in a status between 50 and 4.500 for 100.000 inhabitants per year. Thinking only 
those that they present information of encephalic injury (posttraumatic loss of knowledge, amnesia or others), the average is from 200 to 300 , for 100.000 inhabitants per year. The information published in the USA ranges between 132 (Maryland) and 367 (Chicago) for 100.000 inhabitants per year. In Great Britain between 270 and 430, in France 281, in Norway 200 and in Australia 377, for 100.000 inhabitants per year, respectively.

Of the whole of cranial traumas, between $75 \%$ and $90 \%$ of the cases correspond to light trauma, between $5 \%$ and $25 \%$ to moderate trauma and between $5 \%$ and $20 \%$ to serious trauma, what represents an incidence valuation for 100.000 inhabitants for year from 150 to 270 for the light trauma, from 5 to 60 for the moderate one and from 5 to 50 for seriously, respectively. The incidence of the head injury is much higher in the young people, principally in adults between 15 and 24 years. Most of the series give a $2 / 1$ proportion in favor of the males, in all the age status, except, probably, in the nursing ones. The trauma is more frequent in the ethnic minorities and in the depressed social levels [3]. The labor characteristics are also an important factor, both in the index and in the causal mechanism. If the light cranial traumatism is analyzed only, its incidence increases proportionally in the classes with major socioeconomic power.

\section{Physiopathology of head injury}

Classically concussion has been defined as the momentary loss of brain activity without associated macro or microscopic lesions, but it has now been shown that mild trauma can produce specific histopathological lesions [2]. Experimentation in primates has found a decrease in intracellular free magnesium, a fundamental element in neuronal metabolism, as well as degenerated axons in the brainstem (midbrain and bulge) after a mild trauma. On the other hand, Magnetic Resonance (MR) has shown abnormalities in up to $88 \%$ of patients suffering from mild trauma. As the severity of the trauma increases, the lesions extend to the cerebral hemispheres. In this way, it is necessary to understand head injury as a continuous process and not divided into compartments [4]

\section{Diagnosis and Clinical Management of Head Injury}

In relation to mild trauma, there is much controversy regarding the usefulness of simple skull radiography and the need for hospital observation, in the different literature published so far. From a practical point of view, patients who have suffered mild head injury can be divided into three groups:

\section{Low risk}

Includes patients who meet any of these characteristics: No loss of knowledge or post-traumatic amnesia. Asymptomatic, or discrete headache or dizziness. They may have wound or erosion of soft tissues. In addition, they should not present any characteristic of groups II and III. The incidence of cranial fracture in this group ranges between $0.4 \%$ and $1.6 \%$, and the possibility of developing an intracranial complication is estimated at 1 in 6,000 cases.

\section{Intermediate risk}

Includes the following patients: Loss of knowledge. Post-traumatic amnesia. Progressive headache. Disorientation or alterations of behavior. Vomiting Children under 2 years old. Suspicion of alcohol intake or drug use. Signs of suspected fracture of the base (rhino or otolicuorragia, orbital or retromastoid hematoma). Post-traumatic epileptic crisis. Medical history (alterations of coagulation or anticoagulant therapy, chronic alcoholism, diabetes mellitus, cardiovascular diseases, or others) $[5,6]$.

In this group, the incidence of cranial fracture ranges between 5\% and $10 \%$. If there is a fracture, the risk of complication is estimated to be 60 to 400 times greater than if there is not. In case of complication, the most frequent is the development of contusions and subdural hematomas, with epidural hematomas being rare.

\section{High risk}

Includes patients with any of the following characteristics: Decrease or fluctuation of the level of consciousness not attributable to alcohol, drugs or other causes (metabolic or systemic). Signs of neurological focality. Penetrating wound or palpable depressed fracture. In this group, the possibility of intracranial involvement is high (between 20\% and $70 \%$, depending on the series analyzed) and the patients are candidates for a computerized tomography (CT) of urgency and hospital admission.

The indications for hospital admission for observation in patients in groups I and II are: Alcohol or drug intoxication. Presence of radiological fracture. Previous pathologies (anticoagulation, stroke or others). Disorientation or behavior alterations. Persistent symptoms. Homeless or other social problems. The duration of hospital admission depends on the clinical evolution and the findings in the CT, when it is performed (in the case of fractures, clinical worsening or other causes) [7]. The CT it is the diagnostic method of choice in the acute phase of head injury at the present time. Magnetic Resonance allows detecting lesions difficult to visualize in the CT during the chronic phase of the evolution of head injury, which has made it the diagnostic technique of first choice in this phase. Regardless of the apparent initial severity, in the management of head injury, adequate assessment and treatment of associated trauma is essential.

\section{Prognostic Factors of Head Injury}

Prognostic predictions of disease or trauma have been an important part of medical practice since ancient times. This, although very useful, has many limitations in problems as complex as head injury. The difficulties lie in the large number of variables that influence the outcome, in the relatively long recovery time after a head injury and in the gaps in the knowledge of the physiopathology of the same and its subsequent evolution process [2]. However, there are useful factors in determining the final prognosis, which helps us to make therapeutic decisions and to better understand the physiopathological mechanisms of head injury, such as: age and previous pathologies [6], socioeconomic factors, level of awareness at admission, score in the Glasgow scale, lesions and extra cranial complications, causative mechanisms, radiological findings and laboratory data.

\section{Evolution of Head Injury}

A variety of systems have been used to indicate the evolution of head injury, from mortality rates to sophisticated methods of assessing organic and psychological sequel. Mortality rates are the most used and the most useful, because of their easy determination and because most deaths occur shortly after the trauma, and it is not necessary to analyze long periods of time. It is estimated that up to $60 \%$ of patients with severe trauma die at the accident site. In the hospital setting, the 
first indices published at the beginning of the century gave a $70 \%$ mortality rate for severe trauma, although the figures range from $30 \%$ to $76 \%$, with an average of $40 \%$. Considering only mortality, we do not have a real vision of the problem, because many of the patients who survive are severely affected [8]. The residual defects can be physical (contractures, ossifications), neurological (hemi paresis, seizures, amaurosis), or psychological (alterations of memory, language, behavior). In this regard, it is very important to consider Glasgow's evolutionary scale and neuropsychological tests, despite their respective clinical limitations. The recovery process after a head injury is a dynamic phenomenon and it is important to decide when to assess the final result.

\section{Approach and Objectives}

Cranial traumatism represents one of the main causes of mortality and morbidity in industrialized countries, which constitutes an effort, both at the human and economic levels, very important [1]. The introduction of the CT It was a spectacular advance in the diagnosis of intracranial lesions, allowing us to obtain a very accurate idea of intracranial pathology of traumatic origin [7]. With this diagnostic method it is even possible to induce the existence of lesions not directly evidenced, as is the case of diffuse axonal injury. The CT not only shows the extra or intra-axial major injuries at any time of the evolution, but it allows to acquire dynamic information on the pathological changes that occur at the intracranial level, either spontaneously, or at the request of different medical or surgical therapeutic maneuvers, and certain physiopathological alterations can be demonstrated, such as cerebral edema and intracranial hypertension.

In fact, the follow-up with CT sequential and continuous monitoring of Intracranial Pressure provides knowledge of the intracranial pathophysiology that was unthinkable to have a few years ago, but there are still wide gaps in the understanding of the pathogenesis that limit the development of an optimal therapeutic scheme. In addition, the clinical model of head injury is multifactorial, both clinic and pathological terms and treatment, so it is very difficult to determine the effect of a pathological or therapeutic variable on the evolution or the final prognosis of patients. Currently, it is necessary to determine the most influential factors in the final evolution in order to establish prognostic models and to be able to draw up therapeutic and rehabilitation action plans as effectively as possible. On the other hand, the majority of patients treated for head injury suffer mild trauma; between $1 \%$ and $4 \%$ of these patients will develop life-threatening complications, and a high percentage will show residual symptoms or defects [9]. The Glasgow Coma Scale and the scale introduced by Rimmel et al., which divides trauma into mild, moderate and severe, are universally accepted. The usefulness of these scales, which estimate the severity and prognosis of the trauma, has been proven in the patient suffering from severe trauma, although they are complemented with the assessment of other parameters. However, there are clear discrepancies regarding its application in mild and moderate traumas.

Considering all the previously exposed, this work tries to fulfill the following objectives:

- Bring together a wide range of patients who have suffered mild head trauma and study their epidemiological profile and form of clinical presentation.
- To verify the usefulness and efficacy of the Glasgow Scales for Coma and Rimmel, in determining the severity and prognosis of mild head injury.

- Describe the risk factors that determine the probability of developing intracranial complications in the group of patients suffering from mild trauma and verify the usefulness of simple skull radiography and the need for hospital admission in this group.

- Study the evolutionary patterns in the mild head injury, according to the Jennett and Bond scale, and analyze the different variables that can act on them, determining which have greater prognostic significance.

\section{Criteria of Inclusion and Classification of Patients}

In this work, in which the term traumatism or head injury is used independently of the existence of brain injury or not, all cases of mild head injury have been studied retrospectively, in patients older than 14 years, attended at the University Hospital of Getafe (Madrid, Spain), between December 2010 and December 2015, without any exclusion criteria. The different variables analyzed in patients with mild trauma are: 1.- Age. 2.- Sex. 3.- Background. 4.- Cause of income. 5.- Origin. 6.- Interval trauma-income. 7.- Existence of Neurological Focality on admission. 8.- Score on the Glasgow scale. 9.- Consumption of alcoholdrugs. 10.- Concussion. 11.- Amnesia. 12.- Soft tissue injury. 13.Findings in Radiography. 14.- Findings in CT 15.- Need for Surgery. 16.- Associated injuries. 17.- Income. 18.- Evolution.

\section{Diagnosis and Clinical Management of the Patient with Cranial Trauma}

The patients were divided into 4 groups, according to the score obtained in the Glasgow Scale for Coma, score of 15 and no loss of knowledge or amnesia (Group 1), score of 15 with loss of knowledge and/or amnesia (Group 2), score of 14 (Group 3) and score of 13 (Group 4). In addition, patients with mild trauma were classified as low, intermediate, or high risk, according to the scheme previously reported in the Introduction chapter. In low-risk patients, simple skull radiography was left to medical criteria. If the radiograph showed no injuries, the patient was sent to his home with a recommendation for surveillance for 48 hours. If there was a fracture, CT and the patients remained admitted for 6 to 8 hours for observation. Skull radiography was performed on all patients at intermediate risk, being admitted for observation for at least 8 hours. If after that time the symptoms improved, the patient was referred for home observation. If there was a fracture or the symptoms persisted, CT between 6 and 8 hours after admission, or earlier if there was neurological deterioration of the patient who required it. All high-risk patients have been managed with $\mathrm{CT}$ initial. When the CT detected intracranial injury, in patients of any of the three groups, they entered the Neurosurgery Service, where they have been managed with venous catheterization and application of oxygen therapy by mask, with time controls of their score on the scale of Glasgow, blood pressure and heart rate.

Daily hematological and biochemical routine analyzes were performed during the first 3 days. The patients who needed surgery remained for 24 hours in the Post-Anesthesia Resuscitation Service, being subsequently transferred to Neurosurgery or to the ICU, according to their clinical status. The CT It has been carried out in two different units, in the Emergency Service of the University Hospital of Getafe. The standard scan comprises axial cuts with a separation of 10 
$\mathrm{mm}$. CT of control routinely after any surgical intervention or changes in the patient's situation, and with intervals of 24 to 72 hours, during the first week, in all cases, even when there were no clinical changes that required it. Monitoring of ICP in all patients who showed a focal lesion or diffuse swelling that made the development of intracranial hypertension probable. The figures of ICP they have been measured with intraventricular catheter in right frontal horn or intraparenchymatous sensor, connected to monitor of the Camino type.

The calibration of the base pressure, in the case of the intraventricular technique, was performed against the atmospheric pressure taking as reference the Lundberg point, which corresponds to the roof of the frontal horn of the lateral ventricle. The pressure signal is written on paper and stored in a computer coupled to the monitor that allows to know the minimum, maximum and average pressure during a specific period, and to design the pressure class frequency histogram. Analyzes and cultures of L.C.R. daily to discover infection data early. If there was intracranial hypertension (P.I.C maintained above $20 \mathrm{mmHg}$ ), the following measures were taken: I.- Elevation of the head $15^{\circ}$ to $30^{\circ}$ on the horizontal plane. II.- Control of body temperature with drugs and physical means. III.- Hyperventilation. IVAnalgesia and sedorrelaxation. V.- Ventricular drainage. VI.- Diuretics. VII.- Corticoids. VIII.- Barbiturates.

Considering the general therapeutic actions, all associated metabolic or systemic problems and convulsive states were treated, although routine anticonvulsant therapy was not used, the coagulopathy was solved if it existed, a respiratory physiotherapy treatment and early rehabilitation was initiated, and an adequate nutritional supplement, through parenteral nutrition. The management of focal lesions has followed the guidelines currently set in the various publications included in the medical literature.

\section{Statistical Study of the Sample}

For the descriptive statistics the most usual parameters have been used, such as means, percentages, standard deviations (s), proportions and others. The final evolution of the patient with head injury has been determined at the time of hospital discharge. In mild head injury, everything that is not good recovery, that is, moderate disability, severe disability, persistent vegetative state or death, has been considered an adverse evolution. Within the chapter on analytical statistics, the Chi square test has been used for the bivariate study. The limit of statistical significance has been established in a confidence interval of $95 \%$ $(\mathrm{p}<0.05)$.

\section{Results}

A total of 2,480 patients with mild trauma have been treated. Of these, $1,746(70.4 \%)$ obtain a score of 15 on the Glasgow Scale for the Coma and do not present knowledge loss or amnesia (Group 1), 605 (24.4\%) have a score of 15 with loss of knowledge. knowledge and/or amnesia (Group 2), 86 (3.5\%) score of 14 (Group 3) and $43(1.7 \%)$ score of 13 (Group 4). Following the classification scheme that divides the patients according to risk factors, collected in the Introduction chapter, we have 1,546 low risk patients (62.3\%), 830 intermediate risk (33.5\%) and 104 risk patients high (4.2\%).

In Group 1 there are 2 patients suffering from neurological deterioration $(0.1 \%)$ and 2 suffering from extra neurological deterioration $(0.1 \%)$. In Group 2 there are 2 patients with neurological $(0.3 \%)$ and 1 extra neurological (0.2\%). In Group 3, 2 patients suffered neurological deterioration (2.3\%) and 8 extra neurological (9.3\%). In Group 4, 6 patients suffered neurological deterioration (13.9\%) and 4 extra neurological deterioration (9.3\%). Among patients belonging to Group 1, there are 1,740 cases of good recovery (99.6\%), 3 deaths $(0.2 \%)$ and in 3 the evolution is unknown (0.2\%). In Group 2, 597 patients have good recovery (98.7\%), 1 develops severe disability $(0.2 \%), 3$ die $(0.5 \%)$ and in 4 it is unknown (0.7\%). In Group 3, there are 77 cases of good recovery (89.5\%), 1 moderate disability (1.2\%), 2 severe disabilities (2.3\%), 3 deaths $(3.5 \%)$ and in 3 patients the evolution is unknown (3.5\%). In Group 4, 34 presented good evolution (79.1\%), 2 developed severe disability (4.6\%), 6 died (13.9\%) and in 1 patient evolution was unknown (2.3\%). Among patients classified as low risk, the incidence of cranial fracture is $0.3 \%$, and there is only 1 patient $(0.06 \%)$ with CT pathological (subarachnoid hemorrhage), which presents good recovery. In intermediate-risk patients, the incidence of cranial fracture is $5.2 \%$, CT pathological are 36 (4.5\%), 14 are operated on (1.7\%) and 6 die (0.7\%). Among high-risk patients, the incidence of cranial fracture is $38.3 \%, 54$ have CT pathological (51.9\%), 16 underwent surgery (15.4\%), 9 died (8.6\%), 1 developed moderate disability $(0.9 \%)$ and 4 severe disability $(3.8 \%)$.

\section{Epidemiology and analytical statistics}

A bivariate study of each and every one of the variables has been carried out, showing the most important results below. The older, the worse the score in the E.G.C. $(\mathrm{p}<0.05)$. The relationship between age and causative mechanism is significant $(\mathrm{p}<0.001)$. Among vehicle occupants there are 144 under 20s (19.5\%), 384 have between 20 and 35 (52.2\%), 103 between 36 and 50 (14.1\%), 91 between 51 and 70 $(12,3 \%)$ and 13 are over 70 years old (1.7\%). Among the patients who suffer outrage, 23 are under $20(21.9 \%), 28$ have between 20 and 35 (26.6\%), 17 between 36 and 50 (16.1\%), 23 between 51 and 70 (21.9\%) and 14 are over 70 years old (13.3\%). Among patients suffering direct impact, 105 are under 20 years old (21.9\%), 218 have between 20 and $35(45.5 \%), 79$ between 36 and 50 (16.4\%), 67 between 51 and 70 (13.9\%) and 10 are over 70 years old $(2.1 \%)$. Among patients who suffer a fall, 86 are under 20 (11.3\%), 180 have between 20 and 35 (23.7\%), 99 between 36 and 50 (13.1\%), 189 between 51 and 70 (24.9\%) and 203 are over 70 years old $(26.7 \%)$. The relationship between age and evolution is also significant $(\mathrm{p}<0.001)$. As the age increases, the prognosis is worse. Older patients are more likely to develop intracranial lesions, especially subdural hematomas and contusions, while younger patients more frequently present with diffuse axonal injury and epidural hematoma $(\mathrm{p}<0.001)$. Patients with a medical history are more likely to develop intracranial complications, especially if there are coagulation disorders and a history of chronic alcoholism $(p<0.001)$. Of the 11 CT performed in patients with coagulation disorders, 6 are pathological (54.5\%), and of the 13 performed in chronic alcoholic patients, 12 are abnormal (92.3\%). Patients with a medical history also have a worse evolution $(\mathrm{p}<0.001)$. Among the 12 patients with coagulation disorders there are 4 deaths (33.3\%), and among the 33 alcoholics, 1 dies (3.1\%), 2 develop severe disability (6.2\%) and 1 moderate disability $(3,1 \%)$. The causative mechanisms and the findings in the CT they are significantly related $(\mathrm{p}<0.001)$. Of the $45 \mathrm{CT}$ practiced to vehicle occupants, 19 are pathological $(42.2 \%)$. Of the 13 performed on traumatized patients, 7 are abnormal (53.8\%).

Falls and collisions, more frequent in the elderly, cause subdural hematomas and contusions more frequently than the accident suffered by vehicle occupants, which occurs more often in young people, causing diffuse axonal injury and epidural hematoma. In addition, 
patients who suffer fall and run over have a worse prognosis than the latter, with patients suffering direct impact having the best evolution $(\mathrm{p}<0.001)$. Patients with a focal neurological examination are more likely to develop intracranial complications and worse final prognosis $(\mathrm{p}<0.001)$. Among the 25 patients with focality there are 19 with CT pathological (76\%), 6 deaths (24\%) and 3 with severe disability (12\%), and of the 2,455 without focality there are 55 patients with CT pathological $(2.2 \%), 9$ die $(0.3 \%), 2$ develop severe disability $(0.08 \%)$ and 1 moderate disability $(0.04 \%)$. Associated systemic traumatic injuries are associated with a worse prognosis $(\mathrm{p}<0.001)$. Among the 150 patients with associated trauma (6.1\% of the total), $8(5.3 \%)$ did not show good recovery. The consumption of alcohol and drugs has a relationship that almost becomes significant with evolution $(\mathrm{p}<0.1)$. Of the 193 patients with a history of alcohol ingestion or drug use, 6 do not have a good recovery (3.1\%). Ethyl intake predominates among patients who suffer a traffic accident $(\mathrm{p}<0.01)$. The relationship between the radiological findings and the presence of a scalp wound is not significant, nor is the relationship between it and the final evolution. The existence of fracture is significantly related $(\mathrm{p}<0.01)$ to the causative mechanisms, and thus the patients who suffer fall and run over have a higher incidence of cranial fracture. The presence of cranial fracture is significantly related to the loss of knowledge $(\mathrm{p}<0.01)$, although not with the duration of it. Of the 1,719 patients with normal radiography, 453 presented loss of consciousness (26.3\%). Of the 50 with linear fracture on plain skull radiography, 33 suffer loss of consciousness (66\%), and of the 12 with fracture sinking, 6 have loss of consciousness (50\%).

The findings on plain radiography are also significantly related to the existence of post-traumatic amnesia $(p<0.01)$, but not to the duration of the same. Of the 1,719 patients with normal radiography, 562 suffer from amnesia (32.6\%). Of the 50 with lineal fracture, 38 present amnesia (76\%), and of the 12 patients with sinking fracture, 6 suffer post-traumatic amnesia (50\%). The presence of fracture in simple skull radiography is significantly related to the findings in the CT $(\mathrm{p}<0.01)$. Of the 1,719 patients with normal radiographs, CT to 88 (5.5\%), being pathological in 27 (1.6\% of the total of patients). Of the 50 patients with linear fracture, 31 show CT pathological (62\%). The findings in simple radiology are significantly related to evolution $(p<0.01)$. Of the patients with normal radiography, 5 died $(0.3 \%)$ Among patients with simple fracture, 3 die (6\%), and of patients with fracture sinking, 1 dies (8.3\%). The loss of consciousness is significantly related to the findings in the CT $(p<0.05)$. Of the 92 patients with CT Normal, 36 have lost consciousness (39\%), and among the 91 patients with CT pathological, 54 suffer loss of knowledge $(59.3 \%)$. Loss of consciousness and evolution correlate significantly $(\mathrm{p}<0.05)$. Of the 2,448 patients with good evolution, 536 suffer from loss of knowledge (21.8\%), and among the 21 who do not show good recovery, 11 have lost consciousness (52.4\%). The existence of post-traumatic amnesia is also significantly related to the findings in the CT $(\mathrm{p}<0.05)$. Of the 92 patients with CT Normal, 37 suffer from amnesia (40\%), and among the 91 patients with CT pathological, 55 presented post-traumatic amnesia $(60.5 \%)$. Evolution and posttraumatic amnesia correlate with each other $(\mathrm{p}<0.05)$.

Of the 2,448 patients who have a good evolution, 669 have amnesia (27.3\%), and of the 21 who do not have good recovery, 11 suffer posttraumatic amnesia (52.4\%). The findings in the CT they are significantly related to evolution $(\mathrm{p}<0.001)$. Of the 91 patients with CT pathological, 12 die (13.2\%), 3 develop severe disability (3.3\%) and 1 moderate disability (1.1\%). Among the 92 patients with CT normal, 1 dies $(1 \%)$ and 1 develops severe disability (1\%). The relationship between the score obtained by the patient in the Glasgow Coma Scale and the evolution is variable. Patients in Groups 1 and 2 do not have significant differences in the final evolution. On the contrary, there is a significant difference between their evolution and that of patients in Groups 3 and $4(\mathrm{p}<0.001)$. In turn, patients in Groups 3 and 4 have an evolution that does not become significantly different $(\mathrm{p}<0.1)$. However, there are significant differences $(\mathrm{p}<0.001)$ between low, intermediate and high-risk groups, both in terms of the findings in plain radiography and in $\mathrm{CT}$, and in evolution. There is a significant relationship between the need for surgical indication and evolution $(\mathrm{p}<0.001)$. Of the 30 patients who intervene, $4(13.4 \%)$ have an adverse evolution (moderate or severe disability, or death). In contrast, of the 2,450 patients who are not operated on, only $17(0.7 \%)$ have an unfavorable outcome. Clinical deterioration and evolution are significantly related $(p<0.001)$. Of the 2,440 patients who do not deteriorate, only 3 do not have good recovery $(0.1 \%)$. Of the 12 patients suffering from neurological deterioration, 10 did not show good recovery (83.3\%), and of the 15 who suffer from extra neurological deterioration, $8(53.3 \%)$ do not have a good recovery.

\section{Discussion}

\section{Epidemiological and clinical profile}

Recognizing that trauma is currently the main cause of death in people under 45 years of age, and that $50 \%$ to $75 \%$ of accident deaths are due to head trauma, has led to the acceptance that trauma Cranial nerve represents a public health problem of the highest magnitude $[1,2,9]$. Efforts to reduce mortality have obviously focused on severe trauma, where, despite the advanced diagnostic techniques (Computed tomography and magnetic resonance imaging) [7] and intracranial pressure monitoring, and advances in treatment, there has been no achieved a great decrease in morbidity and mortality rates, mainly due to the fact that the prognosis is closely linked to the brain damage that the patient already presents at the time of care (hence the enormous importance of the use of seat belts, air bags and helmets, to reduce traumatic damage). Therefore, it seems logical to think that efforts should now be directed towards the traumas that are initially considered mild or moderate, in an attempt to minimize brain damage secondary to intracranial complications, and to reduce the morbidity derived from mild uncomplicated traumas. However, these are not easy to study because being so frequent, their attention depends on very diverse medical disciplines. The actual incidence of mild trauma is difficult to determine, for two fundamental reasons. The first is that there are no uniform criteria for the definition of mild head injury, and the second, that not all patients who suffer this type of process are reflected in the care statistics. Different authors consider all head injuries treated in an emergency department as mild for this reason, regardless of whether or not there has been a loss of knowledge, while others only include patients with symptoms of brain injury or those who require hospital admission $[10,11]$.

The incidence of serious neurological complications in patients who are not admitted in the first place is very low but excluding them from the studies carries the risk of not being able to determine what additional tests should be performed in order to detect patients who may subsequently develop neurological complications. It is estimated that only between $15 \%$ and $20 \%$ of all patients suffering head injury require hospital admission. Currently, all patients with a score on the Glasgow Coma Scale of 8 or less tend to be included in the category of severe head trauma, after the application of non-surgical resuscitation 
measures, and those that deteriorate to that level score in the first 48 hours immediately following the trauma. Therefore, a patient with head injury that is initially classified as mild or moderate, and that subsequently deteriorates, must strictly be included among the serious traumas, and its prognosis is similar to that of the latter. To classify patients who suffer mild trauma exclusively on the basis of the Glasgow Scale is to ignore other factors that can trigger a neurological deterioration and condition the final evolution. For this reason, several authors consider that every patient has suffered moderate trauma, with scores of 15 or 14 , requires more than 48 hours, has CT pathological [11] or need neurosurgical intervention, and also all patients with a score of 13. Analyzing the mild head injury, it is necessary to emphasize first the great overload of patients who support the Emergency Services of our environment, due in part to the incorrect functioning of the medicine primary, with the enormous effort in both human and economic terms that it represents. The incidence of mild trauma is higher in young people, with an average age close to 30 years. In our series, which does not include pediatric patients, the average age is higher. Although mild trauma is more frequent in young people, falls predominate in patients older than 65 years, which has already been previously indicated in the literature [9]. The distribution by sex resembles that collected by other authors, with a higher proportion of women than in groups of moderate or severe trauma. As in the industrialized countries, the most common cause of the mild trauma is the traffic accident, with a significant percentage of patients with a history of alcohol intake or drug use, and with a proportion of associated systemic traumatic injuries that increases as the neurological state of the patient worsens.

Traffic accidents are often followed by falls and direct impacts. Due to the broad inclusion criteria of patients employed in our series, the incidence of loss of knowledge and post-traumatic amnesia in patients with a score of 15 on the Glasgow Scale is lower than that reported in the literature, and also therefore, the incidence of cranial fracture among patients with a score of 15 is low, increasing its proportion as the score on the Glasgow Scale worsens. The cranial fracture is more frequent in patients who suffer fall or run over than in patients involved in traffic accidents, as has been demonstrated by other authors. The indication of CT in patients with scores of 14 and 13 it is clearly admitted, the high proportions of intracranial pathology and the need for surgical intervention in patients who obtain 13 points, advise to include the latter in the group of moderate head trauma. However, there are discrepancies regarding the indication of the CT in patients with a score of 15 . Several authors use it routinely, and others only if there is loss of consciousness or post-traumatic amnesia, if there is a cranial fracture, if the patient has an abnormal mental state, or if focal signs are seen, headache, vomiting or ill-defined history. Our series proves the usefulness of CT in the detection of intracranial pathology in these patients [11]. Among young patients, epidural hematoma and diffuse axonal injury predominate, with subdural hematoma and more frequent contusions in elderly patients, as described previously in the literature.

\section{Prognostic Factors}

In the evolution of mild trauma, the significant difference between patients who obtain a score of 15 and those who have 14 points is due to the broad definition of mild head trauma considered in this study. However, there are no differences between the patients who obtain 14 and those who have 13, with the exception of the need for surgical treatment. This raises the question of whether a modification of the
Glasgow Coma Scale is justified. Some authors have proposed a sub classification in patients with a score of 15 , based on the assessment of the degree of orientation in relation to person, time and space, and the mental state of the patient [12].

Patients who score 15 can be divided into three groups, which are those that present some degree of disorientation, those that are oriented, but have behavioral alterations (drowsiness, aggression or other) and are not attentive, and those that have completely normal mental state and are well oriented. The problem arises when determining which criteria should be applied and what kind of examinations should be carried out to carry out this classification [13]. For this, the Galveston Test for Orientation and Amnesia has been developed, which assesses many different parameters. Although this test can be very useful in later evolutionary studies, it is difficult to apply immediately after the trauma, and its interpretation is complex. To simplify the problem, a method of Assessing Mild Alterations of Mental Status has been introduced, which assesses 25 parameters of both cognitive type (orientation, calculation) and activity measurement (spontaneous movements, ability to eat, attention given to the explorer), and that have subsequently been reduced to 12 , which contain the fundamental information [14]. In an attempt to further facilitate the work, we have selected the 4 that were most influential in a recent clinical study, which are the answer to the call, the orientation in person, the orientation in time and the spontaneous language, whose usefulness remains pending verification. Our series demonstrates that the division of patients according to risk groups is very useful in predicting the probability of developing intracranial complications, and therefore in the determination of the evolutionary prognosis of the patient suffering mild trauma. Classification into groups of low, intermediate and high risk is very practical at the time of indicating the need for simple skull radiography, CT cerebral or hospital admission. Different authors have demonstrated the utility of simple skull radiography in mild trauma.

The probability of presenting intracranial complications increases enormously in patients with fracture and altered level of consciousness. If, in addition, the fracture crosses over a vascular groove or a venous sinus, or is located in the cranial base, the incidence of intracranial involvement is even greater. However, in the United States, where light traumas are initially treated by neurosurgeons, skull radiography is not routinely performed, because they believe that it does not modify the management of the patient suffering from mild trauma. In our series, the presence of a cranial fracture is very determinant of the probability of developing intracranial complications and, consequently, of the final prognosis of the patient suffering from mild head injury. The final results in the evolution of the patient with mild trauma are similar to those published in the literature. The most influential prognostic factors in the adverse evolution of mild trauma are the existence of clinical deterioration, the presence of neurological focality, the pathological findings in the $\mathrm{CT}$, the patient's previous medical history and advanced age. The incidence of clinical deterioration increases as the score on the Glasgow Coma Scale obtained by the patient decreases, as does the presence of focal neurological examination. The deterioration is due to extra-neurological causes, pulmonary infection with respiratory insufficiency fundamentally, in more than half of the patients. Alterations in coagulation are the medical background that most influences the evolution of the patient, suggesting that patients with coagulopathies or anticoagulant therapy should be monitored more closely. Several authors also find a higher incidence of late hematomas in this group. Patients with a history of chronic alcoholism also have a greater number of intracranial pathologies, although these 
are less decisive in the final evolutionary outcome. The incidence of complications and mortality in the group of elderly patients is high, and the average hospital stay is higher than that of other patients. Therefore, special attention must be paid to any elderly person suffering from head injury [15]. The most influential variables in the probability of developing intracranial complications in the patient with mild trauma are the presence of neurological focality, the existence of pathological medical history, the advanced age, the abuses and falls as causative mechanisms, the presence of fracture in the radiography simple, loss of knowledge and post-traumatic amnesia. The existence of loss of knowledge and post-traumatic amnesia leads to a higher probability of cranial fracture and pathology on $\mathrm{CT}$, although there is no relationship between the duration of radiological findings and radiological findings, as has been demonstrated by different authors $[2,7,9]$. Because only few parameters (such as neurological deterioration, cranial fracture or hemorrhagic lesions and previous pathological history) appear in the various multivariable studies analyzed as decisive in the appearance of lesions on $\mathrm{CT}$, in the patient suffering from mild trauma, the reliability of them is $92 \%$. It is necessary to indicate the failure obtained in the attempt to find models whose reliability exceeds $95 \%$, as it has been collected in the literature. Therefore, the probability of developing intracranial complications cannot be totally ruled out in patients who suffer loss of consciousness or post-traumatic amnesia, despite having a score of 15 on the Glasgow Coma Scale $[1,10,15]$. However, the incidence of need for surgical intervention in these patients is small.

\section{Conclusion}

Mild head injury is more frequent in males than in females (1.7 / 1), predominantly in patients whose ages range between 20 and 35 years. The most frequent cause is the traffic accident (36.3\%), followed by the fall (35.2\%), the direct impact (22.3\%) and the outrage (5.2\%).

In the clinical management of mild head injury, patients can be classified into groups, according to the risk factors they present. Among low-risk patients, the incidence of cranial fracture is $0.3 \%$, with $0.06 \%$ of CT pathological and $100 \%$ good recovery. In this group, simple skull radiography is of limited use, and patients can be sent for home surveillance without hospital admission. Among patients at intermediate risk, the incidence of cranial fracture is 5.2\%, with $4.5 \%$ of CT pathological and $0.7 \%$ adverse evolution. In these patients, simple skull radiography should be performed, as well as proceed to hospital admission for observation. If there is a cranial fracture or the symptoms persist 8 hours after admission, it is necessary to perform CT to rule out intracranial pathology. Among high-risk patients, the incidence of head fracture is $38.3 \%$, with $51.9 \%$ of CT pathological and $13.3 \%$ unfavorable evolution. These patients should be studied with CT Immediately after being received at the hospital, proceed to your admission, at ICU if the CT it is pathological, and repeat the CT within the first 48 hours.

The most influential parameters in the appearance of lesions in the $\mathrm{CT}$ in patients suffering from mild head injury are the presence of neurological focality, the patient's age and pathological medical history, especially coagulation alterations, and the existence of simple radiography fracture.

The Rimmel Scale correctly adjusts to the severity of head trauma. The Glasgow Coma Scale is only partially related to the evolution of the patient with mild head injury, because it does not consider agitation or orientation.

\section{References}

1. Nguyen R, Fiest KM, McChesney J, Kwon CS, Jette N, et al. (2016) The International incidence of traumatic brain injury: A systematic review and meta-analysis. Can J Neurol Sci 27: 1-12.

2. Barker-Collo S, Theadom A, Jones K, Feigin VL, Kahan M (2016) Accuracy of an International classification of diseases code surveillance system in the identification of traumatic brain injury. Neuroepidemiology 47: 46-52.

3. Thompson FJ, Hou J, Bose PK (2016) Closed-head TBI model of multiple morbidity. Methods Mol Biol 1462: 521-36.

4. Pülhorn H, Westmoreland L, McMahon C (2016) The management of minor head trauma (GCS 15-13) across a Trauma Network. Br J Neurosurg 30: 536-540.

5. Morrison CA, Gross BW, Cook AD, Estrella L, Gillio M, et al. (2016) An analysis of neurosurgical practice patterns and outcomesfor serious to critical traumatic brain injuries in a mature trauma state. J Trauma Acute Care Surg 80: 755-761

6. Sauter TC, Ziegenhorn S, Ahmad SS, Hautz WE, Ricklin ME, et al. (2016) Age is not associated with intracranial haemorrhage in patients with mild traumatic brain injury and oral anticoagulation. J Negat Results Biomed 15: 12 .

7. Livingston DH, Lavery RF, Passannante MR, Skurnick JH, Baker S, et al. (2000) Emergency department discharge of patients with a negative cranial computed tomography scan after minimal head injury. Ann Surg 232: 126-132.

8. Thiruppathy SP, Muthukumar N (2004) Mild head injury: Revisited. Acta Neurochir (Wien) 146: 1075-1082.

9. Shetty VS, Reis MN, Aulino JM, Berger KL, Broder J, et al. (2016) ACR appropriateness criteria head trauma. J Am Coll Radiol 13: 668-679.

10. Uccella L, Zoia C, Perlasca F, Bongetta D, Codecà R, et al. (2016) Mild traumatic brain injury in patients on long-term anticoagulation therapy: do they really need repeated head CT scan? World Neurosurg 93: 100-103.

11. Stocker R, Letta C (2016) Minor Head Injury- a Silent Epidemic. Praxis (Bern 1994) 105: 569-575.

12. Pearn ML, Niesman IR, Egawa J, Sawada A, Almenar-Queralt A, et al. (2016) Pathophysiology associated with traumatic brain injury: current treatments and potential novel therapeutics. Cell Mol Neurobiol 37: 571-585.

13. Gonschorek AS, Schwenkreis P, Guthke T (2016) Mental disorders after mild traumatic brain injury. Nervenarzt 87: 567-579.

14. Viola-Saltzman M, Musleh C (2016) Traumatic brain injury-induced sleep disorders. Neuropsychiatr Dis Treat 12: 339-348.

15. Scott KL, Strong CA, Gorter B, Donders J (2016) Predictors of postconcussion rehabilitation outcomes at three-month follow-up. Clin Neuropsychol 30: 66-81. 